\title{
ANÁLISE DO COEFICIENTE DE PERFORMANCE DE UM CHILLER DOMÉSTICO OPERANDO COM O R-401A EM REGIME TRANSIENTE
}

\author{
Pedro Samuel Gomes Medeiros \\ Graduando de Engenharia Mecânica da Universidade Federal do Rio Grande do Norte - \\ UFRN, Natal-RN. E-mail: pedaof1@hotmail.com \\ Cleiton Rubens Formiga Barbosa \\ Professor do Departamento de Engenharia Mecânica da Universidade Federal do Rio \\ Grande do Norte - UFRN, Natal-RN. E-mail: cleiton@ufrnet.br
}

\section{RESUMO}

Este artigo apresenta uma análise do coeficiente de performance (COP) de um chiller doméstico quando operado em regime transiente. Os dados foram coletados por instrumentos medidores de pressão e temperatura, instalados em pontos estratégicos no equipamento. A análise do COP foi realizada pelo software CoolPack, onde foi feito também um comparativo entre o COP do ciclo ideal e do ciclo real a partir de gráficos pressão versus entalpia específica. Os resultados mostram que o incremento do COP é devido ao aumento considerável da pressão de evaporação do ciclo e que os valores da taxa de incremento são aproximadamente os mesmos entre o ciclo real e o ideal. Através deste trabalho é possível obter conclusões a respeito do comportamento de um ciclo de refrigeração durante o processo de resfriamento a partir do fluido refrigerante utilizado.

PALAVRAS-CHAVE: Ciclo de Refrigeração a Vapor, Chiller Doméstico, Análise do COP, CoolPack. 


\section{ANÁLISE DO COEFICIENTE DE PERFORMANCE DE UM CHILLER DOMÉSTICO OPERANDO COM O R-401A EM REGIME TRANSIENTE}

\section{INTRODUÇÃO}

Chiller é uma máquina térmica que opera segundo o ciclo de refrigeração a vapor, onde o seu fluido de trabalho remove calor de um determinado meio a partir da mudança de fase. Para realizar este processo, quatro componentes principais são usados: compressor, condensador, dispositivo de expansão e evaporador (figura 01).

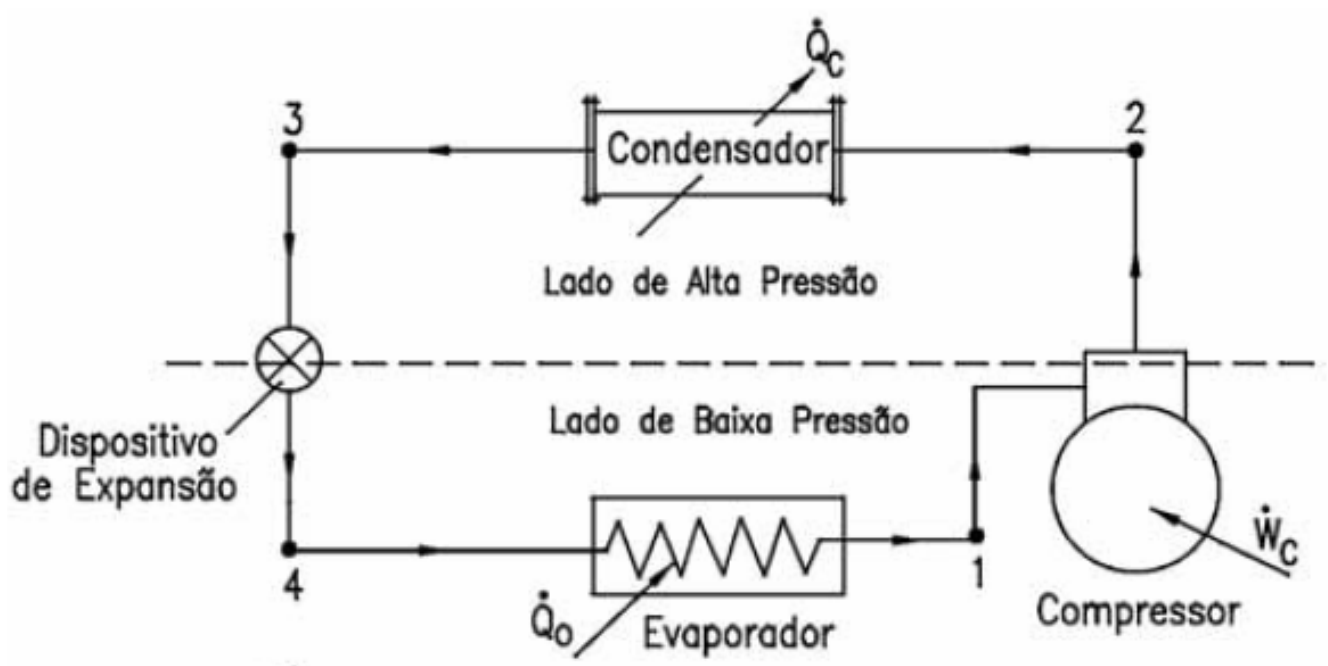

Figura 01: Representação Gráfica de um sistema de refrigeração a vapor (SILVA, 2005)

Os fluidos de trabalho usados em sistemas de refrigeração domésticos (e.g. chiller doméstico) utilizam gases refrigerantes do tipo CFC's (clorofluorcarbono), HCFC's (hidroclorofluorcarbono) e HFC (hidrofluorcarbono). Os gases do tipo CFC reagem com o ozônio, contribuindo para a destruição da ozonosfera, e por esse motivo sua produção e comercialização está banida.

Os gases refrigerantes HCFC e o HFC são hidrocarbonetos parcialmente halogenados e são uma alternativa ao CFC, apresentando pequeno efeito nocivo contra a camada de ozônio (STOECKER; SAIZ JABARDO, 2002). Entretanto, esses refrigerantes contribuem para o aquecimento global, aumentando o efeito estufa. Outros fluidos refrigerantes que estão sendo utilizados atualmente são as misturas de refrigerantes denominados misturas azeotrópicas e misturas zeotrópicas.

As misturas azeotrópicas possuem comportamento similar à das substancias puras durante a mudança de fase de líquido para vapor e vice-versa. As misturas zeotrópicas apresentam comportamento anômalo, pois suas temperaturas de evaporação e de condensação variam durante o processo de mudança de fase, embora mantida as pressões constantes. Essas misturas geralmente têm boas propriedades termodinâmicas e pequeno impacto sobre o ozônio, embora possam contribuir para o efeito estufa. 
Para medir a eficiência de um ciclo de refrigeração a vapor é usado o coeficiente de performance, COP, que é um parâmetro fundamental na análise de sistemas de refrigeração. Relaciona o efeito desejado - refrigeração, com o que se paga por isso energia consumida, avaliando a capacidade do ciclo de retirar calor sobre a potência consumida pelo compressor.

Um ciclo ideal opera dentro da curva de saturação do fluido utilizado, pois assim é possível estabelecer suas propriedades termodinâmicas. Mas, por ser inviavél tecnicamente, o ciclo ideal é modificado a fim de melhorar o desempenho do ciclo, considerando ainda as perdas de carga nos trocadores de calor (condensador e evaporador).

Vários parâmetros influenciam o desempenho do ciclo de refrigeração. Esses parâmetros são, na verdade, modificadores do ciclo a fim de aumentar a sua eficiência. Os fatores são:

- Temperatura de evaporação: sua redução resulta no decréscimo do COP;

- Temperatura de condensação: quanto maior for o seu valor menor será o COP;

- Sub-resfriamento: garante que tenha somente líquido no dispositivo de expansão aumenta a troca térmica no evaporador e consequentemente o COP;

- Superaquecimento: usado para garantir a entrada do fluido no estado gasoso no compressor. Dependendo do gás instalado, pode aumentar ou diminuir o COP.

Na figura 02 é mostrado o gráfico pressão versus entalpia, comparando o ciclo real com o ideal.

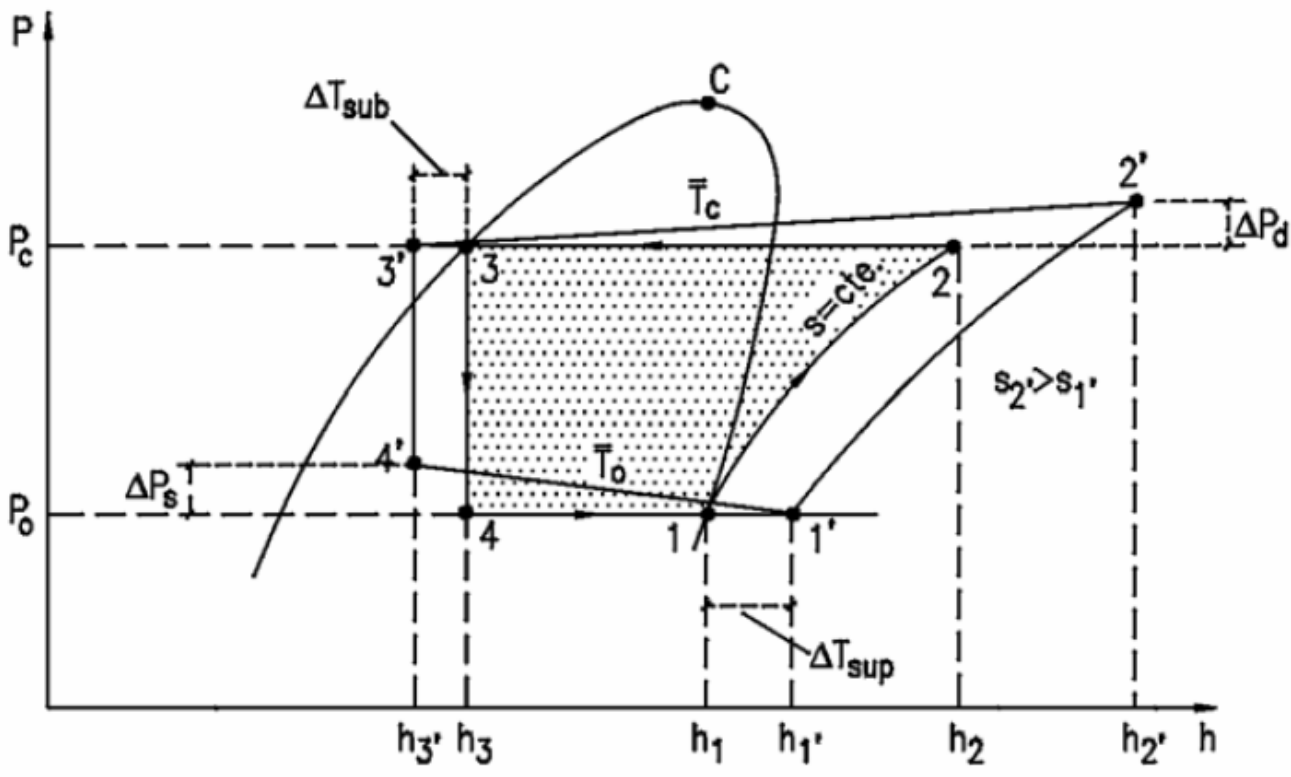

Figura 02: Diferenças entre o ciclo ideal e o real (SILVA, 2005)

A finalidade deste trabalho consiste em analisar o coeficiente de performance do ciclo de refrigeração por compressão a vapor de um chiller doméstico operando com o gás R-401a. Foi verificado o seu comportamento em regime transiente durante o processo de resfriamento. 


\section{METODOLOGIA}

Para determinação experimental do coeficiente de performance (COP) de um chiller doméstico (resfriador de água potável) disponível no Laboratório de Energia do Núcleo de Tecnologia Industrial da UFRN foram instalados estrategicamente ao longo do circuito frigorífico, instrumentos para medição de pressão (manômetros) e de temperatura (termopares).

Originalmente, o protótipo de teste era equipado com o gás refrigerante R-12, um CFC que ataca a camada de ozônio. Para os experimentos, o gás refrigerante HCFC R-401a foi inserido no equipamento de teste em substituição ao gás instalado originalmente, sem modificações das características técnicas do refrigerador de teste.

Inicialmente, preparou-se o ambiente para a realização da prática. Verificou-se se todos os instrumentos e equipamentos estavam funcionando corretamente antes da coleta de dados para avaliação da performance do equipamento. Alguns dos instrumentos empregados na experiência são: manômetros de alta pressão e de baixa pressão, fabricante Gitta (figura 03); controlador digital de temperatura, fabricante Every Control (figura 04); indicadores digitais de temperatura, fabricante Equitherm; e termopares do tipo K (figura 05).

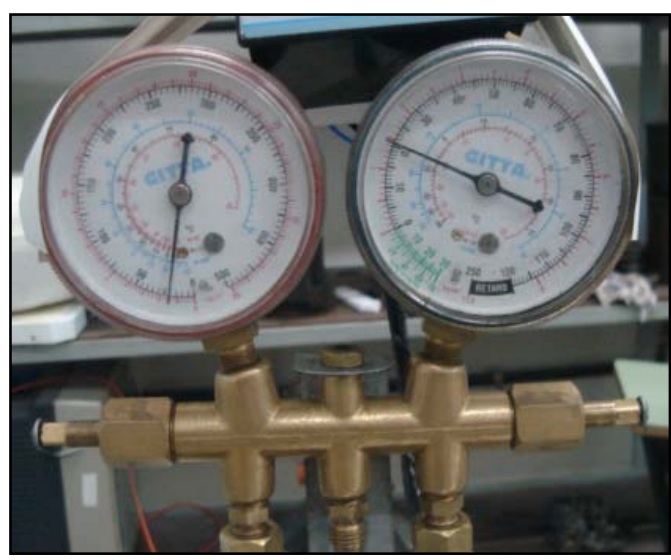

Figura 03: Manômetros de alta e baixa pressão

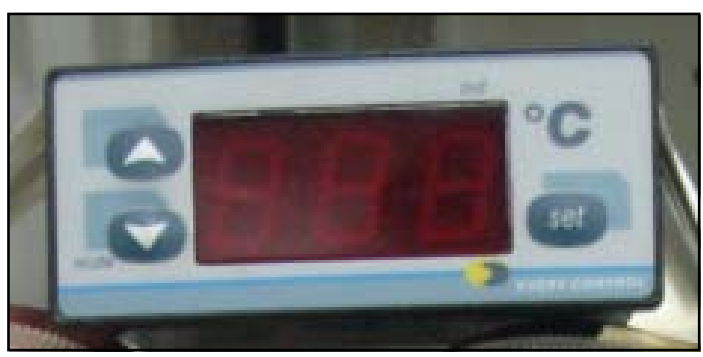

Figura 04: Controlador digital de temperatura 

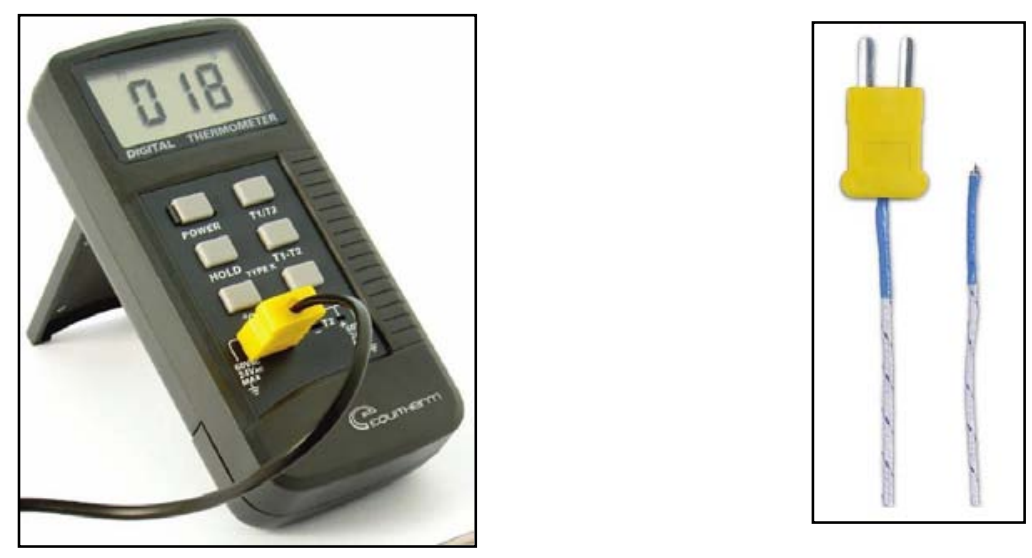

Figura 05: Indicador digital de temperatura e termopar

Os manômetros de alta e de baixa estavam localizados respectivamente depois da saída do compressor e após o tubo capilar. As medições referentes às pressões foram realizadas nestes instrumentos. $\mathrm{O}$ controlador digital de temperatura estava ligado ao reservatório refrigerado medindo a sua temperatura, onde o setpoint foi ajustado em $10{ }^{\circ} \mathrm{C}$. Os termopares eram em número de cinco. Dois foram instalados na entrada e saída do evaporador, dois na entrada e saída do condensador, e um para medição da temperatura ambiente. As leituras das temperaturas foram realizadas com o auxílio do indicador digital de temperatura.

Após a instalação de todos os instrumentos de medição no protótipo de teste, o refrigerador de líquido foi ligado, monitorando-se as leituras de todos os instrumentos, dentro de um intervalo de tempo especificado, e registrando-as em tabela própria.

Foram realizadas quatro leituras das temperaturas de entrada e saída tanto do evaporador como do condensador, pressão de alta e baixa do sistema, temperatura do reservatório indicado pelo controlador digital, e a temperatura ambiente. A primeira medição foi realizada no momento inicial $\left(\mathrm{t}_{0}\right)$, a segunda medição após 15 minutos de funcionamento, a terceira após 30 minutos, e a quarta após 40 minutos quando o controlador digital indicava os $10^{\circ} \mathrm{C}$ ajustados previamente, momentos antes do compressor desligar.

Os dados coletados foram plotados em gráficos com auxílio do software CoolPack, para os cálculos referentes ao COP, transferência de calor do evaporador e do condensador, e a plotagem de gráficos pressão versus entalpia específica (P x h), para serem analisados. 


\section{ANÁLISE DOS RESULTADOS}

Partindo da metodologia já apresentada, os dados colhidos foram examinados no software CoolPack, onde para cada instante foram realizadas as análises do ciclo real e do ciclo ideal, a partir do gráfico pressão versus entalpia específica.

Os dados coletados a cada instante foram: temperatura ambiente, temperatura do reservatório, temperatura na saída do evaporador $\left(\mathrm{T}_{1}\right)$, temperatura na entrada do condensador $\left(T_{2}\right)$, temperatura na saída do condensador $\left(T_{3}\right)$, temperatura na entrada do evaporador $\left(\mathrm{T}_{4}\right)$, pressão de condensação e pressão de evaporação do sistema. Nas tabelas 1, 2 e 3 são mostrados os dados coletados:

Tabela 1 - Temperatura Ambiente e do reservatório

\begin{tabular}{|c|c|c|}
\hline Instante & Temp. Ambiente & Temp. do reservatório \\
\hline Inicial & $25^{\circ} \mathrm{C}$ & $20^{\circ} \mathrm{C}$ \\
15 minutos & $24^{\circ} \mathrm{C}$ & $17^{\circ} \mathrm{C}$ \\
30 minutos & $22{ }^{\circ} \mathrm{C}$ & $12^{\circ} \mathrm{C}$ \\
40 minutos & $21^{\circ} \mathrm{C}$ & $10^{\circ} \mathrm{C}$ \\
\hline
\end{tabular}

Tabela 2 - Temperatura de entrada e saída do evaporador e condensador

\begin{tabular}{|c|c|c|c|c|}
\hline Instante & $\mathbf{T}_{\mathbf{1}}-\mathbf{S e}$ & $\mathbf{T}_{\mathbf{2}}-\mathbf{E c}$ & $\mathbf{T}_{\mathbf{3}}-\mathbf{S c}$ & $\mathbf{T}_{\mathbf{4}}-\mathbf{E e}$ \\
\hline Inicial & $23,2^{\circ} \mathrm{C}$ & $50,3{ }^{\circ} \mathrm{C}$ & $36,5^{\circ} \mathrm{C}$ & $3,9{ }^{\circ} \mathrm{C}$ \\
15 minutos & $20,9^{\circ} \mathrm{C}$ & $58,6^{\circ} \mathrm{C}$ & $37,6{ }^{\circ} \mathrm{C}$ & $3,5^{\circ} \mathrm{C}$ \\
30 minutos & $17,8^{\circ} \mathrm{C}$ & $64,2{ }^{\circ} \mathrm{C}$ & $38{ }^{\circ} \mathrm{C}$ & $2,8^{\circ} \mathrm{C}$ \\
40 minutos & $16,7^{\circ} \mathrm{C}$ & $67{ }^{\circ} \mathrm{C}$ & $39,3{ }^{\circ} \mathrm{C}$ & $2,2{ }^{\circ} \mathrm{C}$ \\
\hline
\end{tabular}

Tabela 3 - Pressão do sistema

\begin{tabular}{|c|c|c|}
\hline Instante & $\begin{array}{c}\text { Pressão de } \\
\text { Condensação }\end{array}$ & $\begin{array}{c}\text { Pressão de } \\
\text { Evaporação }\end{array}$ \\
\hline Inicial & 175 PSI & 5 PSI \\
15 minutos & 180 PSI & 6 PSI \\
30 minutos & 190 PSI & 8 PSI \\
40 minutos & 195 PSI & 10 PSI \\
\hline
\end{tabular}

Para plotar o diagrama termodinâmico $\mathrm{P}$ x h, o software CoolPack solicita as temperaturas de saída do compressor (entrada do condensador $-\mathrm{T}_{2}$ ); a de sub-resfriamento (saída do 
condensador $-\mathrm{T}_{3}$ ); e a de superaquecimento (saída do evaporador $-\mathrm{T}_{1}$ ). A temperatura da saída do compressor é considerada, pois no processo real o processo de compressão não é isentrópico, fator considerado no programa para determinar o COP real.

Para a análise do COP, foram plotados os gráficos do ciclo real e ciclo ideal para cada instante e depois comparados. O COP do ciclo ideal é feito somente com os dados de pressão de condensação e de evaporação; enquanto o ciclo real é realizado com essas pressões e mais as variáveis do processo (superaquecimento, o sub-resfriamento, temperatura do gás na saída do compressor). Na tabela 04, é mostrado os valores do COP, o calor rejeitado pelo condensador $\left(\mathrm{q}_{\mathrm{c}}\right)$, o calor absorvido pelo evaporador $\left(\mathrm{q}_{\mathrm{e}}\right)$, e o trabalho do compressor (w) para cada instante e tipo de ciclo.

Tabela 4 - Análise dos Ciclos em função do Tempo

\begin{tabular}{|c|c|c|c|c|c|}
\hline Tempo & Ciclo & $\mathbf{C O P}$ & $\mathbf{q}_{\mathbf{e}}(\mathbf{k J} / \mathbf{k g})$ & $\mathbf{q}_{\mathbf{c}}(\mathbf{k J} / \mathbf{k g})$ & $\mathbf{w}(\mathbf{k J} / \mathbf{k g})$ \\
\hline \multirow{3}{*}{ Inicial } & Real & 1,63 & 180,49 & 184,98 & 111,62 \\
& Teórico & 1,47 & 125,53 & 211,51 & 85,98 \\
& Real & 1,70 & 177,24 & 190,17 & 105,15 \\
15 minutos & Teórico & 1,54 & 125,78 & 207,91 & 82,13 \\
& Real & 1,84 & 174,28 & 193,53 & 94,96 \\
\multirow{3}{*}{30 minutos } & Teórico & 1,65 & 125,68 & 202,08 & 76,40 \\
& Real & 1,97 & 171,50 & 193,71 & 87,18 \\
40 minutos & Teórico & 1,77 & 126,70 & 198,14 & 71,45 \\
\hline
\end{tabular}

Em todas as análises, percebe-se inicialmente que nos ciclos reais o COP é maior que nos ciclos ideais para cada instante. E ainda, para cada instante há um valor para o COP, sendo que o mesmo aumenta com o passar do tempo atingindo o maior valor momentos antes do compressor desligar, quando o setpoint de $10^{\circ} \mathrm{C}$ iria ser alcançado.

Como pode ser visto, com o passar do tempo a pressão de evaporação teve um incremento considerado. As pressões foram aumentando com o tempo, assim como também a capacidade de refrigeração do sistema. Como consequência, o COP do refrigerador também aumentou. Como o COP cresce quando há incremento da pressão de evaporação e decremento da pressão de condensação, vê-se que após 40 minutos a pressão de evaporação teve um aumento de $100 \%$, enquanto que a pressão de condensação incrementou apenas $11,43 \%$.

A temperatura ambiente foi diminuindo e o calor rejeitado $\left(\mathrm{q}_{\mathrm{c}}\right)$ foi aumentando, melhorando a capacidade de troca térmica do condensador. A pressão de todo o sistema foi aumentando, diminuindo o trabalho do compressor. Todas essas variáveis de processo influenciaram no resultado do COP, fazendo que o mesmo aumentasse. Essa é a justificativa para a melhora da capacidade de refrigeração do ciclo quando passados 40 minutos de funcionamento. 
Analisando a evolução do COP (gráfico 01), verifica-se que a taxa de incremento entre o ciclo real e o ciclo ideal é aproximadamente constante durante todo o processo. A taxa média durante o experimento é de $11 \%$. Isso quer dizer que as modificações introduzidas no ciclo real do chiller aumentam o rendimento em $11 \%$ quando comparado com o ciclo ideal. Os incrementos do COP real em relação ao ideal são mostrados na tabela 05:

Tabela 05 - Incremento do COP do ciclo real em relação ao ideal

\begin{tabular}{|c|c|c|c|}
\hline Instante & Ciclo & COP & $\begin{array}{l}\text { Incremento do } \\
\text { COP real ao idea }\end{array}$ \\
\hline \multirow[b]{2}{*}{ Inicial } & Real & 1,63 & \multirow[b]{2}{*}{$10,9 \%$} \\
\hline & Teórico & 1,47 & \\
\hline \multirow[b]{2}{*}{15 minutos } & Real & 1,70 & \multirow[b]{2}{*}{$10,4 \%$} \\
\hline & Teórico & 1,54 & \\
\hline \multirow[b]{2}{*}{30 minutos } & Real & 1,84 & \multirow[b]{2}{*}{$11,5 \%$} \\
\hline & Teórico & 1,65 & \\
\hline \multirow{2}{*}{40 minutos } & Real & 1,97 & \multirow{2}{*}{$11,3 \%$} \\
\hline & Teórico & 1,77 & \\
\hline
\end{tabular}

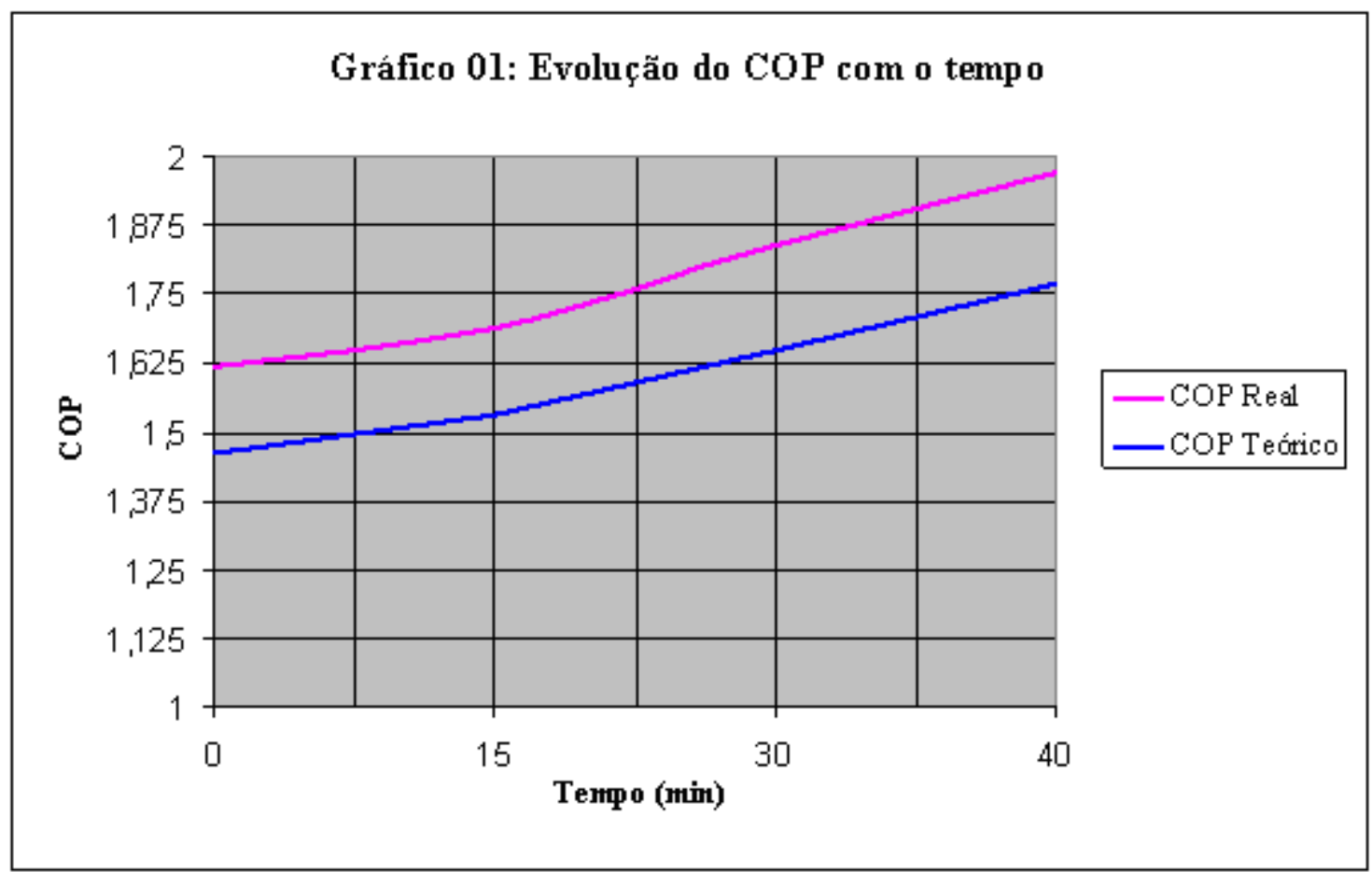

Os gráficos 02, 03, 04 e 05 mostram os ciclos reais e ideais para cada instante analisado em gráficos do tipo pressão versus entalpia específica: 


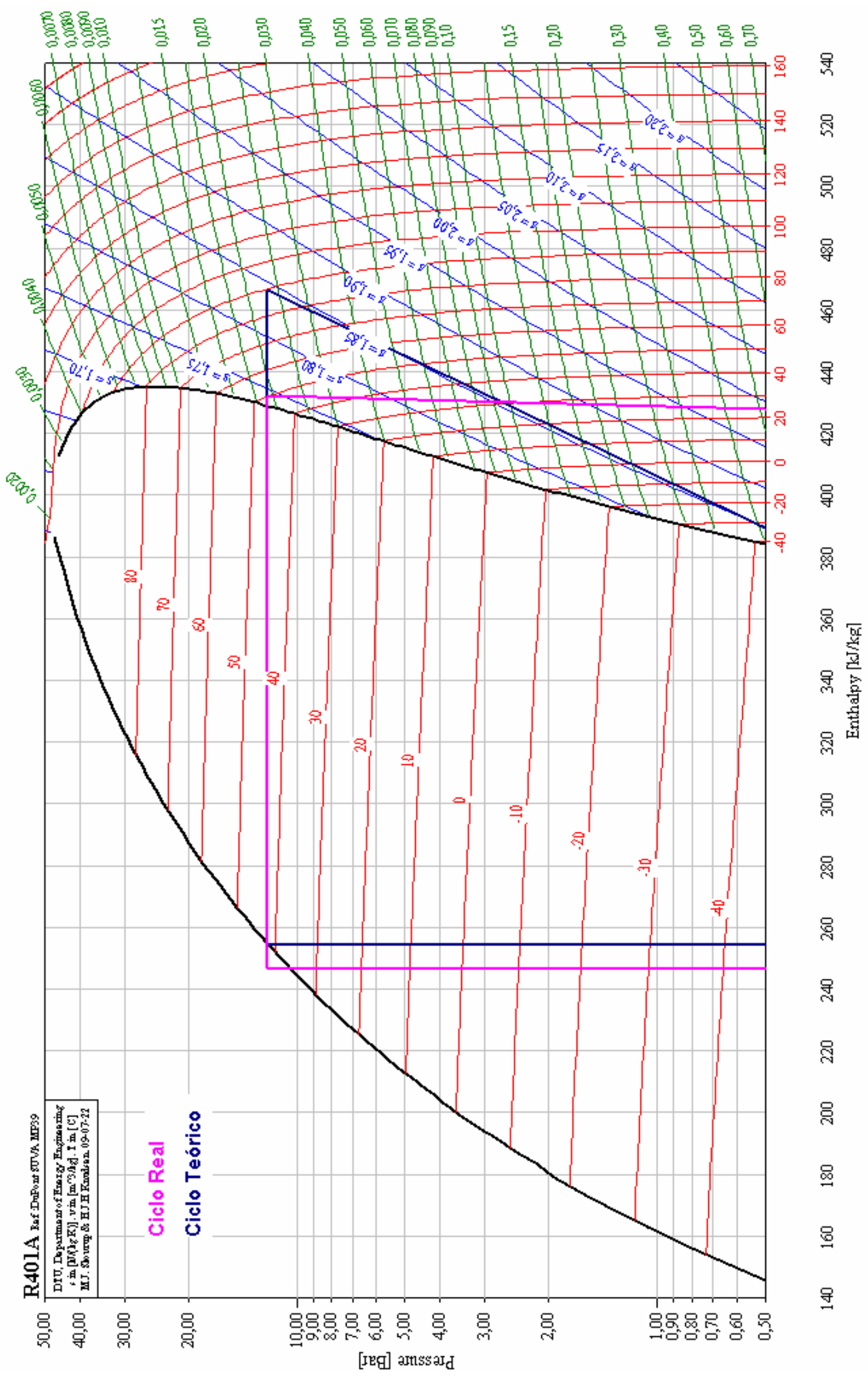

Gráfico 02: Comparação entre os ciclos Real e Teórico (ideal) - Inicial 


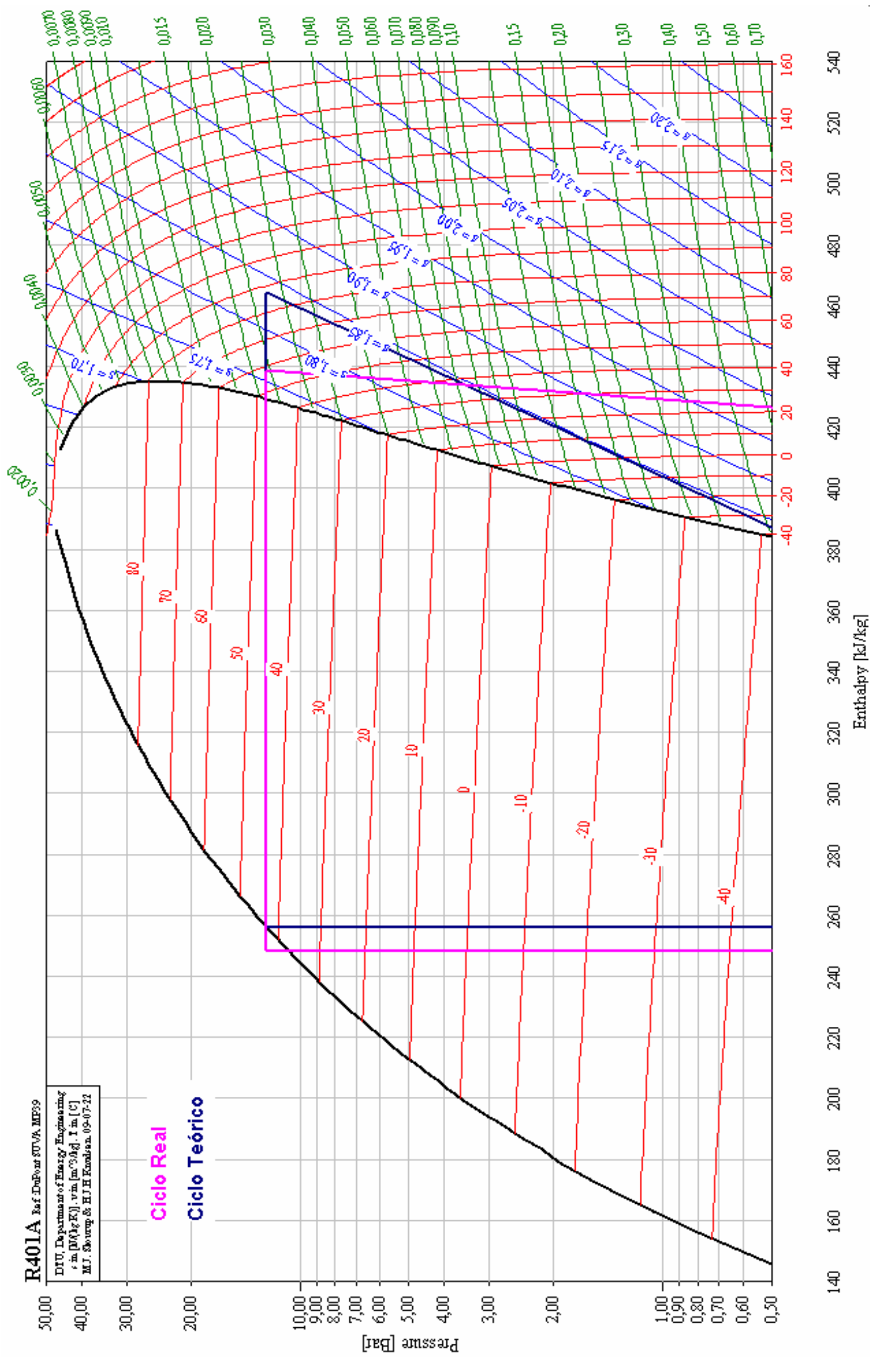

Gráfico 03: Comparação entre os ciclos Real e Teórico (ideal) - 15 Minutos 


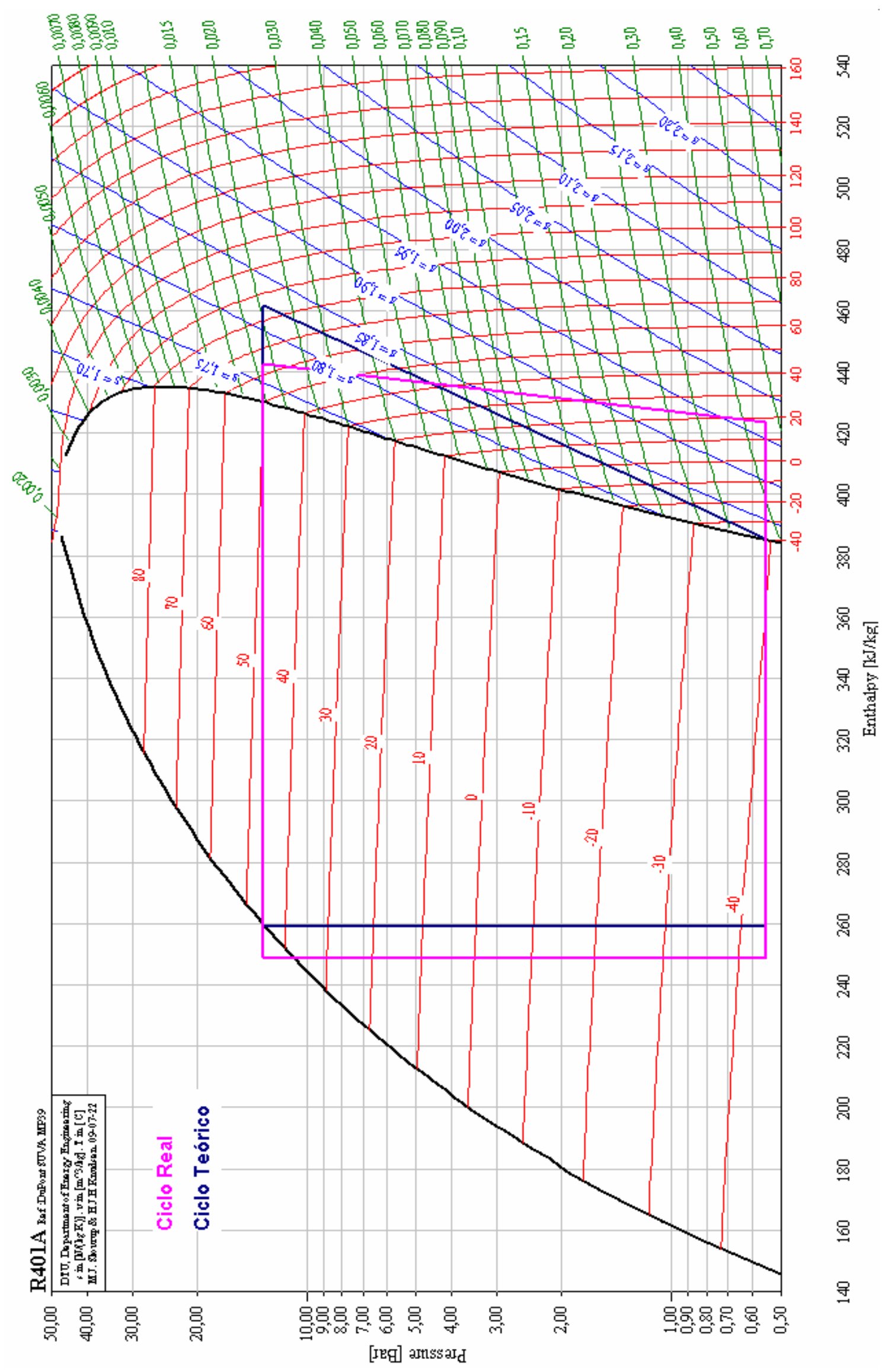

Gráfico 04: Comparação entre os ciclos Real e Teórico (ideal) - 30 Minutos 


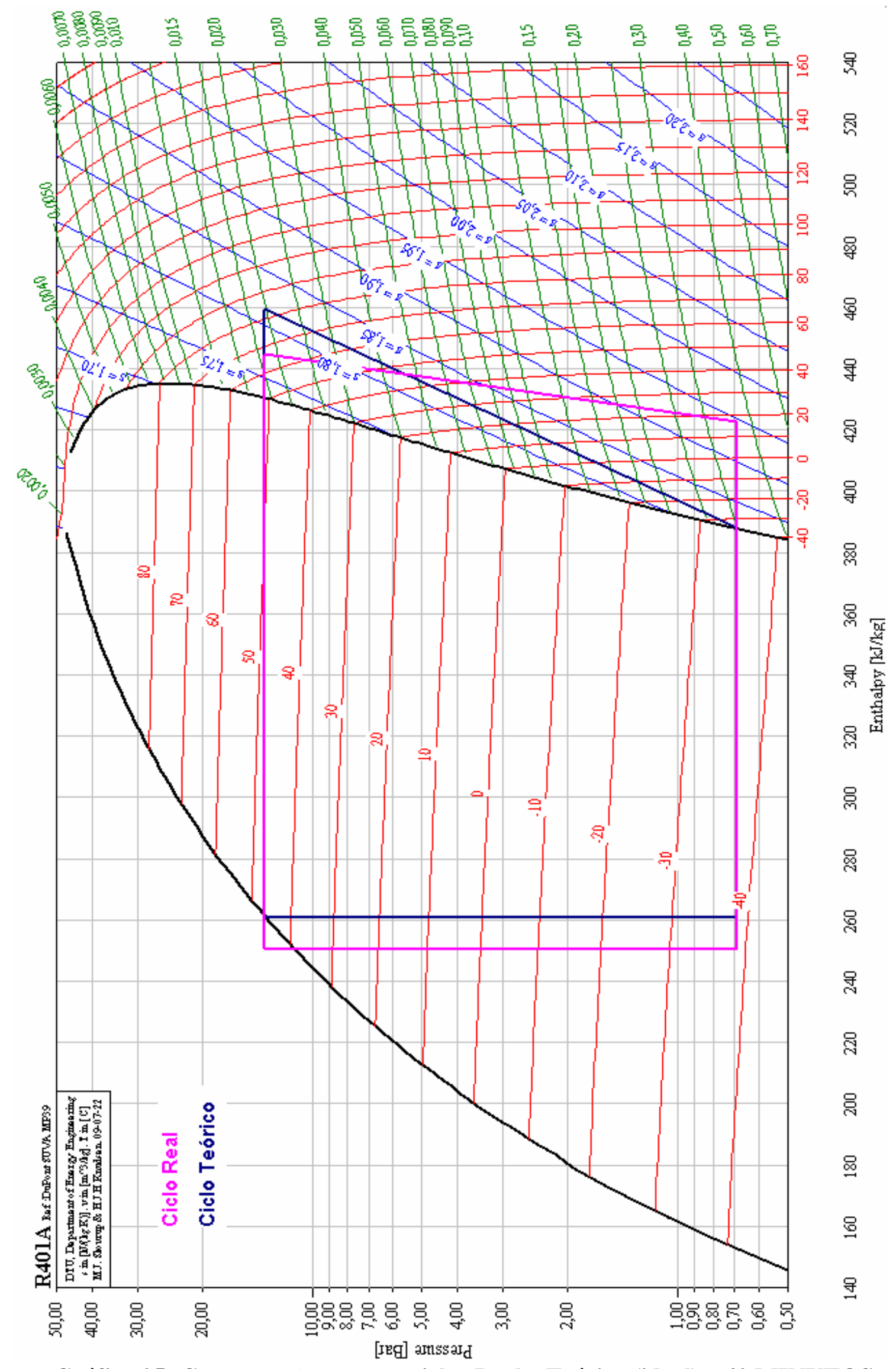

Gráfico 05: Comparação entre os ciclos Real e Teórico (ideal) - 40 MINUTOS 
Comparando os gráficos do ciclo real com o ciclo teórico, percebe-se inicialmente que a área do gráfico do ciclo real é maior, revelando que há uma quantidade maior de calor sendo trocado, influenciando mais uma vez no COP. Outro detalhe é que na saída do condensador, o fluido no ciclo teórico está na linha de saturação com título igual a zero, e na saída do evaporador o fluido está também na linha de saturação, com título igual a um.

No ciclo real, o sub-resfriamento e o superaquecimento servem não apenas para melhorar a eficiência do ciclo, mas também, para garantir respectivamente que na válvula de expansão tenha somente líquido, e que na entrada do compressor tenha somente gás.

\section{CONCLUSÕES}

Vimos que os ciclos ideais de refrigeração não são aplicados rigorosamente em sistemas reais de refrigeração. Há considerações físicas, como a expansão isoentálpica da válvula de expansão e a compressão isoentrópica do compressor, que no ciclo real não podem existir por limitações técnicas. Mesmo assim, é possível ter ciclos reais operando com as mesmas pressões de um ciclo ideal com uma eficiência térmica melhor.

Somente com a análise no software CoolPack, percebemos que o COP de um sistema térmico não é constante num processo transiente e na medida em que a pressão de evaporação incrementa, aumenta a capacidade de refrigeração, desde que esse incremento seja bem superior ao incremento de pressão de condensação.

As modificações do ciclo com o sub-resfriamento e o superaquecimento são sempre aplicadas a fim de melhorar a eficiência do ciclo e garantir um funcionamento adequado de todo o sistema.

\section{AGRADECIMENTOS}

Ao Laboratório de Energia, do Núcleo de Tecnologia Industrial da UFRN.

\section{REFERÊNCIAS BIBLIOGRÁFICAS}

MIHAI, I.; MILICI, D.; CRASI, M. Experimental Determination Of Temperature In One Stage Vapor Compression Refrigeration Cycle. Annals of the Oradea University. Fascicle of Management and Technological Engineering, Volume VII (XVII), 2008.

SILVA, M. N. da. Eficiência Energética em Sistemas de Refrigeração Industrial e Comercial. Rio de Janeiro: Eletrobrás, 2005.

STOECKER, W. F.; SAIZ JABARDO, J. M. Refrigeração Industrial. 2a edição. São Paulo: Edgard Blücher, 2002. 